\title{
Water Particle Trajectory and Mass Transport of Internal Waves Propagating Over A Constant Slope
}

\author{
Kim-Cuong NGUYEN ${ }^{1}$, Motohiko UMEYAMA ${ }^{2}$, Tetsuya SHINTANI ${ }^{3}$ \\ ${ }^{1}$ Member, PhD student, Department of Civil and Environmental Engineering \\ Tokyo Metropolitan University (1-1-Minami-Osawa, Hachioji, Tokyo, 192-0397, Japan) \\ ${ }^{1}$ Vietnam National University, Hanoi (334-Nguyen Trai, Thanh Xuan, Hanoi, Vietnam) \\ ${ }^{2}$ Member, Professor, Department of Civil and Environmental Engineering \\ Tokyo Metropolitan University (1-1-Minami-Osawa, Hachioji, Tokyo, 192-0397, Japan) \\ ${ }^{3}$ Member, Assistant Professor, Department of Civil and Environmental Engineering \\ Tokyo Metropolitan University (1-1-Minami-Osawa, Hachioji, Tokyo, 192-0397, Japan)
}

\begin{abstract}
This paper presents experimental and theoretical investigations of water particle trajectory and mass transport of internal waves propagating over a sloping bottom in a two-layer densitystratified water. The water particle trajectory has been obtained by experimental, numerical, and analytical methods for two different thickness ratios as well as two wave periods. In addition, the mass transport velocity was estimated. The trajectory of individual particle is not closed but shows a quasi-elliptic pattern. It was confirmed from the measured and computed results that the particle near the density interface yields a maximum forward drift while one away from interface tends to move backward to conserve the mass in the closed wave tank. The theoretical solution gives reasonable results for the drift of the water particle near the density interface.
\end{abstract}

Key Words: Internal waves, water particle trajectory, mass transport, constant slope

\section{Introduction}

When waves propagate from the deep water to the shallow water, they steepen, deform, and finally break. The water particle behavior during the shoaling event is the key to understand the nonlinear wave mechanics that include the mass transport. The particle motion leads to a net mass transport in the direction of the wave propagation. For surface waves, the mass transport became a well-known physical aspect by the investigation of Longuet-Higgins $(1953)^{1)}$. On the other hand, some solutions for water particle trajectories under the Stokes surface waves have been derived by Constantin $(2006)^{2)}$, Constantin and Villari (2008) ${ }^{3}$, and Chang et al. $(2009)^{4)}$. For internal waves, the water particle trajectory and mass transport are still not well documented although internal waves are believed that they cause the cross-shelf transport and affect the dispersal of larvae, sediment or phytoplanktons. In a stratified fluid of two layers, Wunsch $(1971)^{5)}$ investigated the effects of internal waves that encounter a sloping beach, based upon the Lagrangian velocity. Umeyama and Matsuki (2011) investigated theoretically and experimentally the kinematics of internal waves in a fluid of a constant depth with different thickness ratios of upper (fresh water) and lower (salt water) layers. Later, Umeyama et al. (2012) measured the particle trajectories over a constant slope in the same internal wave tank. Although numerous investigations related to nonlinear internal waves have been undertaken to examine the water particle path and mass transport in a two-layer density-stratified water, no theoretically justifiable solution is available. Moreover, the Lagrangian properties of particle path are still uncertain because of a lack of understanding the behavior of water movement under the sea surface. The purpose of this study is to reveal the water particle trajectory and the mass transport under internal waves propagating above a slope experimentally and theoretically. This investigation extends the previous study and validates the measurements of Umeyama et al. $(2012)^{7)}$ by using a two-dimensional (2D) numerical simulation. We also propose a theoretical solution for predicting water particle trajectory.

The idea of application of the method of characteristics for water particle trajectory in the real beach is presented in section 2. The basics of experimental setups and numerical model are roughly described in sections 3 and 4. Section 5 describes the main results of particle trajectory and mass transport. Finally, conclusions are drawn in section 6 .

\section{Method of characteristics for water particle trajectory}

Consider the internal waves propagating over a uniform slope in a two-layer density-stratified fluid. The origin of the axes is set at the intersection between the undisturbed interface and the slope. Umeyama and Shintani $(2004)^{8)}$ derived a reliable solution for the displacement of the density interface and the horizontal velocity in the lower layer by means of the method of characteristics. The governing equations for the internal 
waves in the lower layer are

$$
\begin{gathered}
2\left(1+\frac{\eta_{I I}+h_{I I}}{h_{I}}\right) C_{t}+u_{I I x} C+2\left(1+\frac{\eta_{I I}+h_{I I}}{h_{I}}\right) C_{x}=0 \\
u_{I I t}+u_{I I} u_{I I x}+2\left(1+\frac{\eta_{I I}+h_{I I}}{h_{I}}\right)^{2} C C_{x}=g \frac{\rho_{I I}-\rho_{I}}{\rho_{I I}} h_{I I x}
\end{gathered}
$$

where $\eta_{I I}=$ interfacial displacement, $h_{I}=$ upper layer thickness, $h_{I I}=$ lower layer thickness, $\rho_{I}=$ upper layer density, $\rho_{I I}=$ lower layer density, $C$ =internal wave celerity, $u_{I I}=$ horizontal velocity in the lower layer, $t=$ time, $x$ =distance from the origin, and $g=$ gravity acceleration.

$$
C=\sqrt{g \frac{\left(\rho_{I I}-\rho_{I}\right)}{\rho_{I I}} \frac{\left(h_{I I}+\eta\right) h_{I}}{h_{I I}+\eta+h_{I}}}
$$

The lower-layer thickness for a uniform slope is given by

$$
h_{I I}=h_{I I}(x)=m \frac{\rho_{I}}{g\left(\rho_{I I}-\rho_{I}\right)} x
$$

where $m=$ a constant. By adding and subtracting Eqs.(1) and (2), and assuming $\eta+h_{I I}<<h_{I}$, the results can be written in the familiar form:

$$
\left[\frac{\partial}{\partial t}+\left(u_{I I} \pm C\right) \frac{\partial}{\partial x}\right]\left(u_{I I} \pm 2 C-m t\right)=0
$$

Use of the method of characteristics will make it possible to describe $\eta_{I I}$ and $u_{I I}$ such as

$$
\begin{aligned}
\eta_{I I}= & -\frac{A^{2}}{2} \frac{h_{I}+s x}{h_{I} s x}\left[J_{1}(X) \cos T+\left\{J_{0}(X)-\frac{J_{1}(X)}{X}\right\} \sin T\right]^{2} \\
+ & A\left\{J_{0}(X) \sin T+J_{1}(X) \cos T\right\} \\
u_{I I}= & A \sqrt{\frac{\rho_{I I}-\rho_{I}}{\rho_{I I}} g \sqrt{\frac{h_{I}+s x}{h_{I} s x}}\left[J_{1}(X) \cos T\right.} \\
& \left.+\left\{J_{0}(X)-\frac{J_{1}(X)}{X}\right\} \sin T\right]
\end{aligned}
$$

where $X=\frac{2 \omega}{s} \sqrt{\frac{h_{I} s x}{h_{I}+s x} / \frac{\rho_{I I}-\rho_{I}}{\rho_{I I}} g}$ and $T=-\omega t$

where $A=$ a constant, $s=$ slope, $\omega=$ angular frequency, and $J_{n}(X)=$ Bessel function of order $n$. Hereafter, the dependent variable $X$ will not be shown.

In the upper layer, the momentum and continuity equations may determine the surface displacement and the horizontal velocity in the same manner. According to Umeyama et al. $(2012)^{7)}$, these are

$$
\begin{aligned}
& u_{I}= \frac{C\left(\eta_{I}-\eta_{I I}\right)}{h_{I}+\left(\eta_{I}-\eta_{I I}\right)} \\
& \eta_{I}=-\frac{A^{2} \sigma C}{g} \int \frac{h_{I}+s x}{h_{I}^{2} s x}\left[J_{1} \sin T-\left\{J_{0}-\frac{J_{1}}{X}\right\} \cos T\right] \\
& \times\left[J_{1} \cos T+\left\{J_{0}-\frac{J_{1}}{X}\right\} \sin T\right] d t \\
& \quad-\frac{A \sigma C}{g h_{I}} \int\left\{J_{0} \cos T-J_{1} \sin T\right\} d t
\end{aligned}
$$

To predict the water particle trajectories of internal waves on a slope, we denote $\left(x_{I}(t), z_{I}(t)\right)$ and $\left(x_{I I}(t), z_{I I}(t)\right)$ the instantaneous water particle positions at time $t$ in the upper and lower layers, respectively. The corresponding velocities are $u_{I}=\partial x_{I} / \partial t$, and $w_{I}=\partial z_{I} / \partial t$ in the upper layer, $u_{I I}=\partial x_{I I} / \partial t$, and $w_{I I}=\partial z_{I I} / \partial t$ in the lower layer. When the mean position of a water particle is given at $\left(\overline{x_{I}}, \overline{z_{I}}\right)$ for the upper layer or at $\left(\overline{x_{I I}}, \overline{z_{I I}}\right)$ for the lower layer, the instantaneous water particle position is denoted as $x_{I}(t)=\overline{x_{I}}+\varsigma_{I}(t)$ and $z_{I}(t)=\overline{z_{I}}+\xi_{I}(t) \quad, \quad$ or $\quad x_{I I}(t)=\overline{x_{I I}}+\varsigma_{I I}(t) \quad$ and $z_{I I}(t)=\overline{z_{I I}}+\xi_{I I}(t)$, where $\varsigma_{I}, \xi_{I}$ and $\varsigma_{I I}, \xi_{I I}$ are the horizontal and vertical displacements in the upper and lower layers, respectively.

Umeyama et al. (2012) ${ }^{7)}$ arrived at the solution of $\varsigma_{I}$ and $\varsigma_{I I}$ as

$$
\begin{aligned}
\varsigma_{I}= & \frac{A^{2} C}{2 \sigma h_{I}} \frac{h_{I}+s x}{h_{I} s x}\left\{-J_{1}^{2}\left(\frac{T}{2}+\frac{\sin 2 T}{4}\right)+J_{1} J_{01} \frac{\cos 2 T}{2}\right. \\
& \left.+J_{01}^{2}\left(-\frac{T}{2}+\frac{\sin 2 T}{4}\right)\right\}-\frac{A C}{\sigma h_{I}}\left(J_{0} \cos T-J_{1} \sin T\right) \\
\varsigma_{I I}= & -\frac{A \varepsilon_{g}}{\sigma} \sqrt{\frac{h_{I}+s x}{h_{I} s x}}\left(J_{1} \sin T+J_{01} \cos T\right) \\
& +\frac{A^{2} \varepsilon_{g}^{2}}{\sigma^{2}}\left(\frac{h_{I}+s x}{h_{I} s x}\right)\left[\frac { 1 } { 2 s x ^ { 2 } } \frac { h _ { I } s x } { h _ { I } + s x } \left(J_{1}^{2} \frac{\cos 2 T}{4}\right.\right. \\
& \left.+J_{1} J_{01} \frac{\sin 2 T}{2}-J_{01}^{2} \frac{\cos 2 T}{4}\right)-J_{1} J_{01} \frac{d X}{d x} \frac{\cos 2 T}{4} \\
& +J_{0}\left\{J_{1} \frac{d X}{d x}-\frac{1}{X}\left(\frac{1}{2 s x^{2}} \frac{h_{I} s x}{h_{I}+s x} J_{1}-J_{01} \frac{d X}{d x}\right)\right\} \\
& \times\left(-\frac{T}{2}+\frac{\sin 2 T}{4}\right)-J_{01}^{2} \frac{d X}{d x}\left(\frac{T}{2}+\frac{\sin 2 T}{4}\right) \\
& -J_{01}\left\{J_{1} \frac{d X}{d x}-\frac{1}{X}\left(\frac{1}{2 s x^{2}} \frac{h_{I} s x}{h_{I}+s x} J_{01} \frac{d X}{d x}\right) \frac{\cos 2 T}{4 \sigma}\right\}
\end{aligned}
$$

where $J_{01}(X)=J_{0}(X)-J_{1}(X) / X$ 


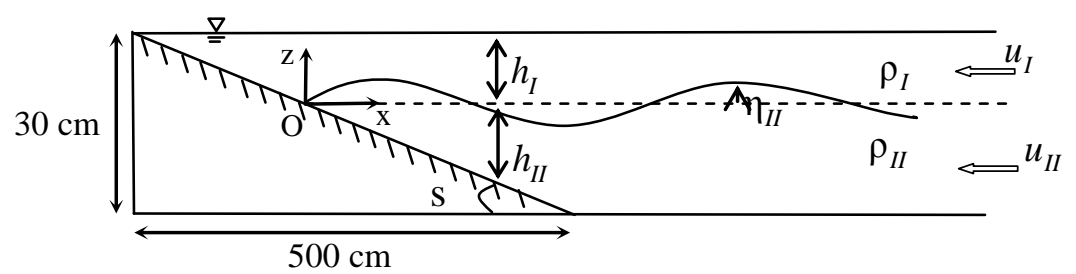

Fig. 1 Sketch of internal waves in the wave tank

\section{Numerical method}

In this section, theoretical aspect for internal waves is discussed with a two dimensional numerical model ${ }^{8)}$. In this 2D model, the Boussinesq approximation is applied to the continuity and momentum equations. The governing equations are

$$
\frac{\partial u}{\partial x}+\frac{\partial w}{\partial z}=0
$$

$\frac{D u}{D t}=-\frac{1}{\rho} \frac{\partial P}{\partial x}+\frac{\partial}{\partial x}\left\{\left(v+v_{t}\right) \frac{\partial u}{\partial x}\right\}$

$+\frac{\partial}{\partial z}\left\{\left(v+v_{t}\right) \frac{\partial u}{\partial z}\right\}$

$\frac{D w}{D t}=-\frac{1}{\rho} \frac{\partial P}{\partial z}-\frac{\rho-\rho_{0}}{\rho_{0}} g+\frac{\partial}{\partial x}\left\{\left(v+v_{t}\right) \frac{\partial w}{\partial x}\right\}$

$+\frac{\partial}{\partial z}\left\{\left(v+v_{t}\right) \frac{\partial w}{\partial z}\right\}$

$\frac{D \rho}{D t}=\frac{\partial}{\partial x}\left\{\left(\frac{v}{S c}+\frac{v_{t}}{\sigma_{t}}\right) \frac{\partial \rho}{\partial x}\right\}+\frac{\partial}{\partial z}\left\{\left(\frac{v}{S c}+\frac{v_{t}}{\sigma_{t}}\right) \frac{\partial \rho}{\partial z}\right\}$

$v_{t}=c_{\mu} \frac{k^{2}}{\varepsilon}$

$\frac{D k}{D t}=\frac{\partial}{\partial x}\left\{\left(v+\frac{v_{t}}{\sigma_{\varepsilon}}\right) \frac{\partial k}{\partial x}\right\}+\frac{\partial}{\partial z}\left\{\left(v+\frac{v_{t}}{\sigma_{\varepsilon}}\right) \frac{\partial k}{\partial z}\right\}$

$+P_{r}+G-\varepsilon$

$\frac{D \varepsilon}{D t}=\frac{\partial}{\partial x}\left\{\left(v+\frac{v_{t}}{\sigma_{\varepsilon}}\right) \frac{\partial \varepsilon}{\partial x}\right\}+\frac{\partial}{\partial z}\left\{\left(v+\frac{v_{t}}{\sigma_{\varepsilon}}\right) \frac{\partial \varepsilon}{\partial z}\right\}$

$+c_{\varepsilon 1} \frac{\varepsilon}{k}\left\{P_{r}+\left(1-c_{\varepsilon 3}\right) G\right\}-c_{\varepsilon 2} \frac{\varepsilon^{2}}{k}$

$P_{r}=v_{t}\left[2\left(\frac{\partial u}{\partial x}\right)^{2}+2\left(\frac{\partial w}{\partial z}\right)^{2}+\left(\frac{\partial u}{\partial x}+\frac{\partial w}{\partial z}\right)^{2}\right]$

$G=g \frac{v_{t}}{\sigma_{t}} \frac{\partial \rho}{\partial z}$ where $w=$ vertical velocity; $P=$ pressure; $v=$ kinematic viscosity; $v_{t}=$ kinematic eddy viscosity; $S c=\mathrm{Schmidt}$ number $\left(=638\right.$ for saline water at $\left.\mathrm{T}=20^{\circ} \mathrm{C}\right) ; \sigma_{t}=$ turbulent Schmidt number $(=1.0) ; k=$ turbulent kinetic energy; $P_{r}=$ turbulent production due to mean train; $G=$ turbulent production due to buoyancy force; $\varepsilon=$ dissipation rate; $c_{\mu}=0.09 ; \sigma_{k}=1.0 ; \sigma_{\varepsilon}=1.3 ; \sigma_{\varepsilon 1}=1.44 ; \sigma_{\varepsilon 2}=1.92 ;$ and $\sigma_{\varepsilon 3}=1.0$. To solve these governing equations, an explicit finite difference technique with the fractionalstep method is employed on staggered grids. The advective terms of Eqs. (12)-(14) and Eqs. (16) and (17) are discretized using the ULTIMATE-QUICKET scheme, while the diffusive terms are discretized by the central difference scheme. With those techniques, the velocity and density distribution are computed in the whole flume with the spatial resolutions of $\Delta x=0.03 \mathrm{~m}$ and $\Delta z=0.005$ $\mathrm{m}$, and time step of $\Delta t=0.005 \mathrm{~s}$. The particle trajectory is estimated with the same technique described above for the experiments.

\section{Experiments}

Experiments were conducted in a wave tank, having an overall length of $600 \mathrm{~cm}$ and a cross section $15 \mathrm{~cm}$ wide by $35 \mathrm{~cm}$ deep. A slide-type wave generator with a Dshaped wave paddle was placed at one end. A 1-cm-thick Plexiglas plate, which served as the plane seabed with slope 3 in 50, was fabricated between 100 and $600 \mathrm{~cm}$ from the wavemaker. A density-stratified fluid consisting of fresh water in the upper layer and salt water in the lower layer was prepared for a series of experiments. The density of the salt water was $1,030 \mathrm{mg} / \mathrm{cm}^{3}$, and the water depth was kept at $30 \mathrm{~cm}$ during all tests. The upper and lower thickness ratio was chosen at $h_{I}: h_{I I}=15 \mathrm{~cm}: 15$ $\mathrm{cm}$, and $10 \mathrm{~cm}: 20 \mathrm{~cm}$. The internal waves with periods of $\mathrm{T}=5.0 \mathrm{~s}$ and $7.2 \mathrm{~s}$ have been generated for both thickness ratios. A 1-cm laser sheet of uniform intensity was emitted from the upper side, and covered an area between the surface and the sloping bed. The system used a high-definition digital video camera with a maximum resolution of 1920 X 1080 pixels. The size of the field of view (FOV) for the PIV measurement was $64 \mathrm{~cm} \times 30$ $\mathrm{cm}$. The water was seeded with DIAION whose mean grain size and specific gravity were $0.11 \mathrm{~mm}$ and 1.01 , respectively. In addition, an internal wave gauge was set at $100 \mathrm{~cm}$ from the wavemaker to measure the variation of the interfacial displacement. The interfacial displacements have been obtained using Laser-Induced - 
Table 1 Measured wave height at $100 \mathrm{~cm}$ from wavemaker for different experimental cases.

\begin{tabular}{|c|c|c|c|}
\hline Exp. & Thickness ratio & Wave period & Wave height \\
\hline 1 & $15 \mathrm{~cm}: 15 \mathrm{~cm}$ & $5.2 \mathrm{~s}$ & $2.6 \mathrm{~cm}$ \\
\hline 2 & $15 \mathrm{~cm}: 15 \mathrm{~cm}$ & $7.2 \mathrm{~s}$ & $2.7 \mathrm{~cm}$ \\
\hline 3 & $10 \mathrm{~cm}: 20 \mathrm{~cm}$ & $5.2 \mathrm{~s}$ & $2.8 \mathrm{~cm}$ \\
\hline 4 & $10 \mathrm{~cm}: 20 \mathrm{~cm}$ & $7.2 \mathrm{~s}$ & $2.4 \mathrm{~cm}$ \\
\hline
\end{tabular}

Fluorescence (LIF) method.

Recently, the PIV technique has been applied to investigate the physical aspects of internal waves ${ }^{910) 11)}$.The basic principle of PIV is evaluating the instantaneous velocities through recording the position of images of small tracers, suspended in the fluid, at successive instants in time. In this investigation, vector fields could be obtained with the PIV system processing a pair of images, using an interrogation window of 64 x 64 pixels in a candidate region of $128 \times 128$ pixels. A crosscorrelation method was performed to calculate the water particle displacement and local velocity by processing a pair of image frames. Although the representation of the velocity vector field in a Eulerian system is a typical example of the PIV method, the result can be applied to a particle tracking process in a Lagrangian system. In general, the particle motion within a tracking time step $\Delta t$ on a Eulerian grid could be estimated as following procedures:

(1) The nodal velocities in a Eulerian system were computed by PIV method.

(2) The particle velocity of a certain location was obtained by interpolating four velocities at neighboring nodal points, and

(3) A particle location was determined by translating the particle with the obtained particle velocity.

\section{Results}

\subsection{Interfacial displacements}

The numerical model is calibrated using the wave height estimated at the location of $100 \mathrm{~cm}$ from the wave maker. After that, the computed interfacial displacement from the 2D hydrodynamic model is compared with one from the LIF measurement. For example, in the case of $h_{I}$ $: h_{I I}=15 \mathrm{~cm}: 15 \mathrm{~cm}$, and $\mathrm{T}=7.2 \mathrm{~s}$, the measured internal wave height is approximately $2.7 \mathrm{~cm}$ at $\mathrm{x}=100 \mathrm{~cm}$ (Table 1). Fig. 2 shows a comparison between the measured and computed interfacial displacements at $x=150 \mathrm{~cm}$. The temporal interfacial variation by the $2 \mathrm{D}$ model is in good agreement with the measured data. Because it is impossible to avoid the effects of return flow, reflection and higher-order harmonies owing to the closed tank in most experiment, the discrepancy between experimental and computed results sometimes occurs. However, this agreement strongly supports the usefulness of the numerical method for internal waves on a sloping bottom. Figs. 3 and $\mathbf{4}$ show the interfacial displacements computed by the Eq. 6 for $h_{I}: h_{I I}=15 \mathrm{~cm}: 15 \mathrm{~cm}$ at $\mathrm{t} / \mathrm{T}=0.25$ with $\mathrm{T}=5.2 \mathrm{~s}$ and $\mathrm{T}=7.2 \mathrm{~s}$, respectively. Umeyama and Shintani $(2004)^{8)}$ confirmed that the meth-

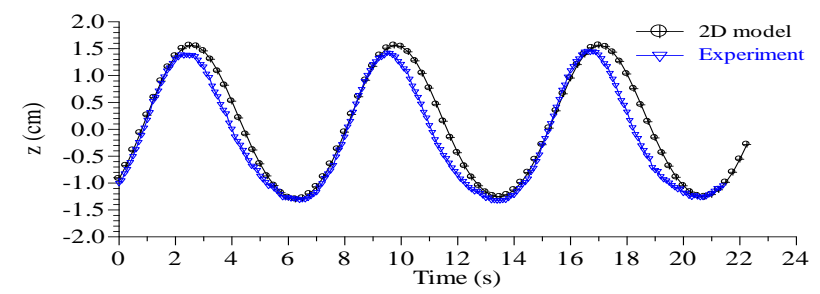

Fig. 2 Interfacial displacements at $x=150 \mathrm{~cm}$ for $\mathrm{h}_{\mathrm{I}}: \mathrm{h}_{\mathrm{II}}=15 \mathrm{~cm}: 15 \mathrm{~cm}$, and $\mathrm{T}=7.2 \mathrm{~s}$ from the $2 \mathrm{D}$ model and the present experiment

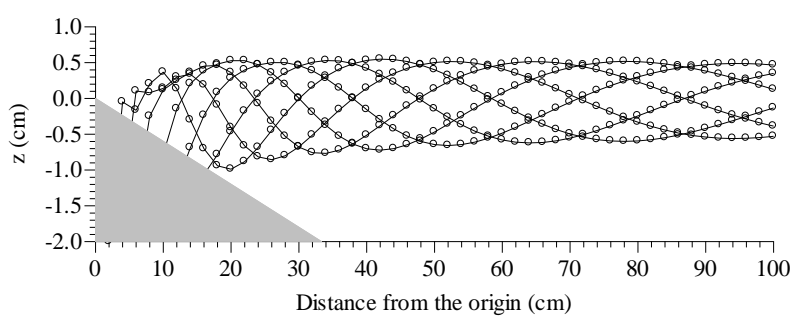

Fig. 3 Computed interfacial displacements for $\mathrm{h}_{\mathrm{I}}: \mathrm{h}_{\mathrm{II}}=15 \mathrm{~cm}: 15 \mathrm{~cm}$, and $\mathrm{T}=5.2 \mathrm{~s}$ by Eq. 6

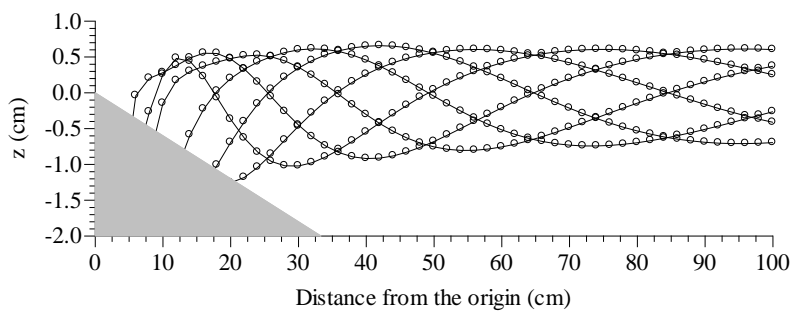

Fig. 4 Computed interfacial displacements for $\mathrm{h}_{\mathrm{I}}: \mathrm{h}_{\mathrm{II}}=15 \mathrm{~cm}: 15 \mathrm{~cm}$, and $\mathrm{T}=7.2 \mathrm{~s}$ by Eq. 6

-od of characteristics can be predict adequately the details of the rump profile of internal waves on the sloping bottom.

\subsection{Water particle trajectory}

Fig. 5 shows the plots of the measured and computed particle orbit geometries for $h_{I}: h_{I I}=15 \mathrm{~cm}: 15 \mathrm{~cm}$ during three wave cycles for $\mathrm{T}=5.2 \mathrm{~s}$. Fig. 5 (a) depicts the water particle trajectories at five elevations at the location of $x=93 \mathrm{~cm}$. The triangle symbol shows a position of water particle estimated from the PIV measurement, and the crossed circle symbol depicts the instantaneous location computed by the numerical model. In the upper layer, the maximum drift distance of measured particle is about 3 $\mathrm{cm}$ while the particle drift near the density interface is approximately $9 \mathrm{~cm}$ during three wave cycles. The computed trajectory by the $2 \mathrm{D}$ model near the interface is similar to the measured profile. At some elevations in the upper layer, the measured particle path is extended further in the direction opposing to the wave propagation when compared to the computed result. In the lower layer, the computed particle paths are in terrible disorder. Near the interface, the measured particle movement shows a similar trend to the computed one despite the 
larger drift distance for the experimental once. Fig. 5 (b) illustrates the water particle trajectories at six elevations at the location of $x=118.5 \mathrm{~cm}$. The water particle moves 3 $\mathrm{cm}$ in the offshore direction in the upper layer and $2 \mathrm{~cm}$ in the shoreward direction in the lower layer. Near the interface, the drift distance of particle is $7 \mathrm{~cm}$ but its rotational direction is opposite to the calculated one. Agreement of PIV data with numerical result supports the existence of the Stokes drift. This result strongly recommends further measures of interfacial displacement in the internal wave tank and calibrated with the numerical model. Water particles move clockwise in the upper layer and anticlockwise in the lower layer. Near the density interface, the horizontal and vertical displacements of the water particle are large relative to those near the surface and bottom regions. The particle marches forward in a large nonclosed loop at the density interface, implying that each particle yields a maximum forward drift.

Fig. 6 shows a comparison of measured and computed particle trajectories at $\mathrm{x}=106$ and $118.5 \mathrm{~cm}$ for $\mathrm{h}_{\mathrm{I}}: \mathrm{h}_{I I}=15$ $\mathrm{cm}: 15 \mathrm{~cm}$ and $\mathrm{T}=7.2 \mathrm{~s}$. The trajectories in the lower and upper layers are predicted well by the numerical model while there is a discrepancy for both particle trajectories near the interface. The measured particle drifts a shorter horizontal excursion compared to that in the lower or upper layer. The computed particles move further in the forward direction near the density interface.

Fig. 7 depicts the water particle trajectories in the case of $h_{I}: h_{I I}=10 \mathrm{~cm}: 20 \mathrm{~cm}$ with $\mathrm{T}=5.2 \mathrm{~s}$. The initial positions of water particle are $x=114 \mathrm{~cm}$ and $169.5 \mathrm{~cm}$. A fair agreement is obtained between experiment and numerical results especially in the pattern of movement. Near the interface at $\mathrm{x}=114 \mathrm{~cm}$, the measured particle moves shoreward about $3 \mathrm{~cm}$ while the computed particle moves about $4 \mathrm{~cm}$. The particles shift in the offshore direction is noticeable in the lower layer. Near the interface at $\mathrm{x}=169.5 \mathrm{~cm}$, the measured particle moves relatively long distance toward the shore. However, there is a discrepancy between the measured and computed trajectories there. All the particle trajectories are not closed in the total depth. The water particles around density interface tend to move in the direction of the wave propagation while particles in the remaining region travel in the opposite direction because of mass conservation in the closed tank.

Fig. 8 illustrates the water particle trajectories for $h_{I}$ : $\mathrm{h}_{\mathrm{II}}=10 \mathrm{~cm}: 20 \mathrm{~cm}$ and $\mathrm{T}=7.2 \mathrm{~s}$. Fig. 8 (a) depicts the trajectories at six elevations at $\mathrm{x}=126 \mathrm{~cm}$. The measured trajectory shows a discrepancy to the computed ones near the interface. In the upper layer, both computed and measured particles move in the shoreward direction while these in the lower layer move to the offshore direction to maintain the mass balance in the closed tank. In this case, the upper layer is thicker than the lower layer so that the particles near the interface were strongly affected by the interfacial displacement. This trend is not the same as one along the slope. Fig. 8 (b) shows the water particle trajectories at $\mathrm{x}=155 \mathrm{~cm}$. At this location, the measured water particles in the upper layer tend to move to the offshore direction while these in the lower layer drift toward the shore. In contrast, the computed particles drift toward the shore in the total lower layer and the offshore in the total upper layer.

Fig. 9 depicts a comparison of the theoretical water particle trajectories estimated by the method of characteristics and measured particle trajectories for $h_{I}$ : $\mathrm{h}_{\mathrm{II}}=15 \mathrm{~cm}: 15 \mathrm{~cm}$ during three wave cycles. Fig. 9 (a) shows the trajectories at $x=93 \mathrm{~cm}$ with $\mathrm{T}=5.2 \mathrm{~s}$, while Fig. 9 (b) shows these at $x=106 \mathrm{~cm}$ with $T=7.2$ s. Fig. 10 illustrates the similar paths for $h_{I}: h_{I I}=10 \mathrm{~cm}: 20 \mathrm{~cm}$. Figs. 10 (a) and (b) show the water particle trajectories at $\mathrm{x}=114 \mathrm{~cm}$ with $\mathrm{T}=5.2 \mathrm{~s}$ and at $\mathrm{x}=155 \mathrm{~cm}$ with $\mathrm{T}=7.2 \mathrm{~s}$, respectively. Comparing the measured particle paths and those computed from the method of characteristics at the same locations, the ideal paths are qualitatively in fair agreement with the measured ones. The discrepancy occurs because the analytical solution has been derived without assuming the reflection on the bottom and the return flow from the upper slope. In addition, the measured data involves higher harmonics generated by the wavemaker. The theoretical prediction is predictable in the region just above and below the density interface. (a) $x=93 \mathrm{~cm}, \mathrm{~h}=20.58 \mathrm{~cm}$

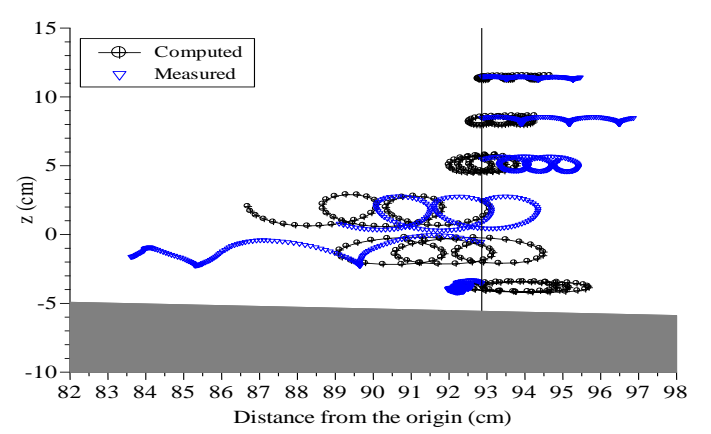

(b) $\mathrm{x}=118.5 \mathrm{~cm}, \mathrm{~h}=22.11 \mathrm{~cm}$

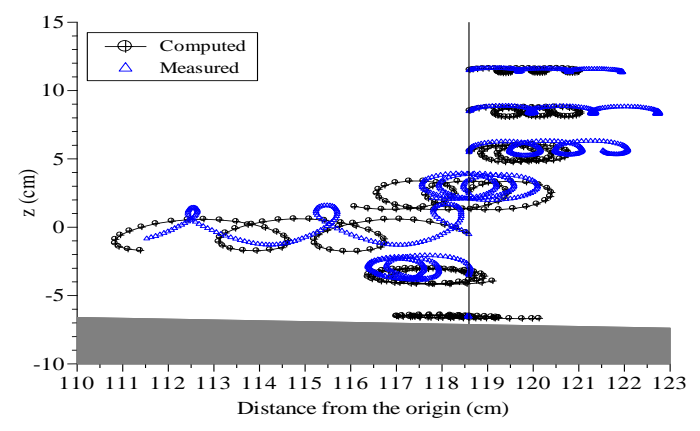

Fig. 5 Water particle trajectories for $h_{I}: h_{I I}=15 \mathrm{~cm}: 15 \mathrm{~cm}$, and $T=5.2 \mathrm{~s}$ 
(a) $\mathrm{x}=106 \mathrm{~cm}, \mathrm{~h}=21.36 \mathrm{~cm}$

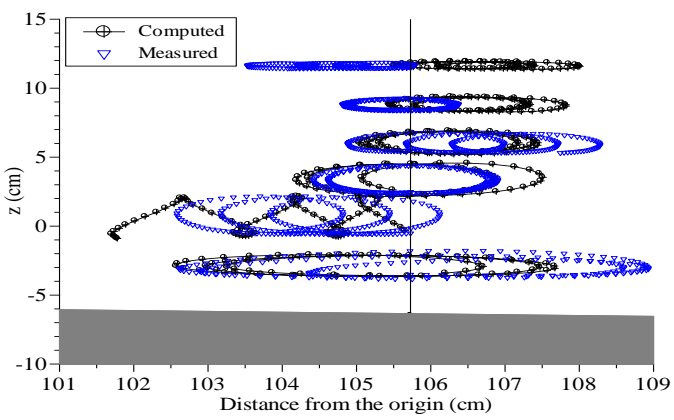

(b) $\mathrm{x}=118.5 \mathrm{~cm}, \mathrm{~h}=22.11 \mathrm{~cm}$

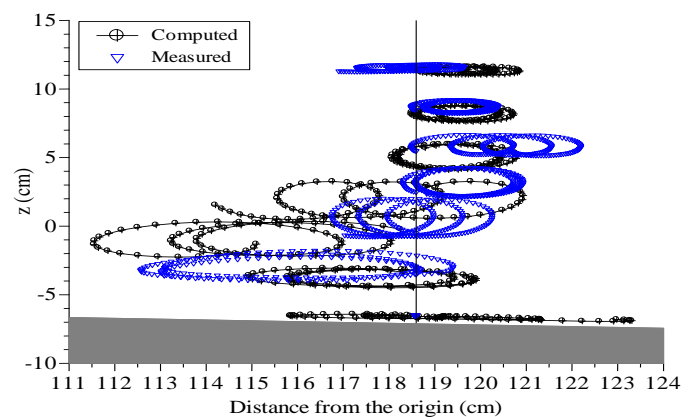

Fig. 6 Water particle trajectories forh $\mathrm{h}_{\mathrm{I}}: \mathrm{h}_{\mathrm{II}}=15 \mathrm{~cm}: 15 \mathrm{~cm}$, and $\mathrm{T}=7.2 \mathrm{~s}$

(a) $\mathrm{x}=114 \mathrm{~cm}, \mathrm{~h}=16.84 \mathrm{~cm}$

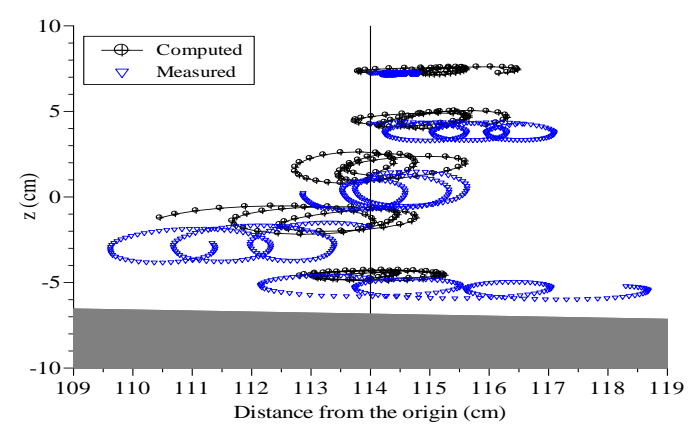

(b) $\mathrm{x}=169.5 \mathrm{~cm}, \mathrm{~h}=20.17 \mathrm{~cm}$

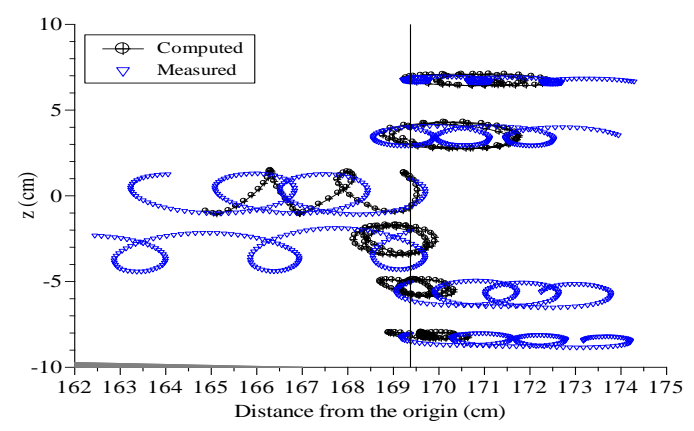

Fig. 7 Water particle trajectories for $h_{I}: h_{I I}=10 \mathrm{~cm}: 20 \mathrm{~cm}$, and $T=5.2 \mathrm{~s}$

(a) $x=126 \mathrm{~cm}, \mathrm{~h}=17.56 \mathrm{~cm}$

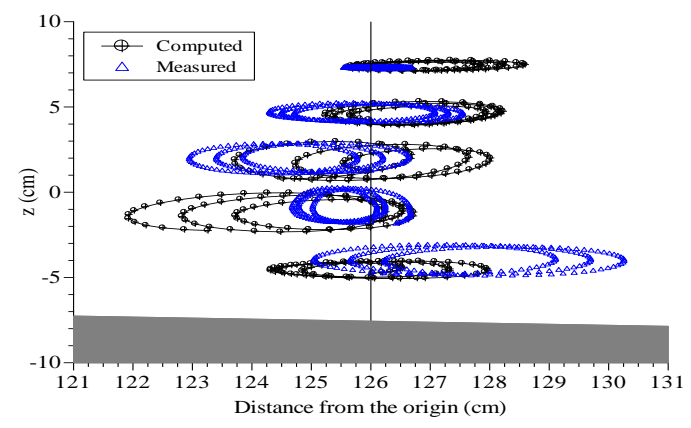

(b) $\mathrm{x}=155 \mathrm{~cm}, \mathrm{~h}=19.3 \mathrm{~cm}$

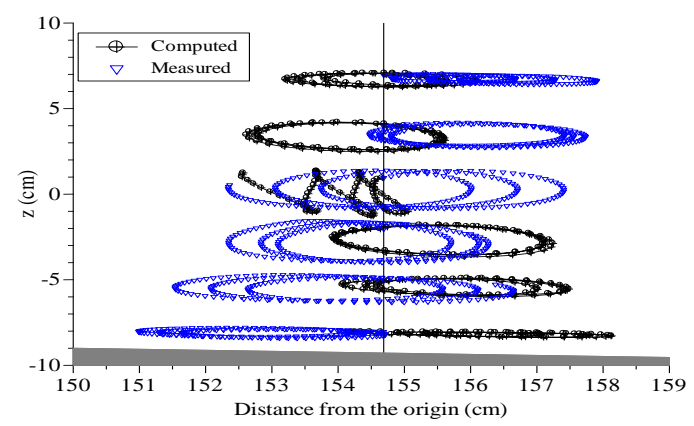

Fig. 8 Water particle trajectories for $h_{I}: h_{I I}=10 \mathrm{~cm}: 20 \mathrm{~cm}$, and $T=7.2 \mathrm{~s}$

(a) $\mathrm{T}=5.2 \mathrm{~s}, \mathrm{x}=93 \mathrm{~cm}$

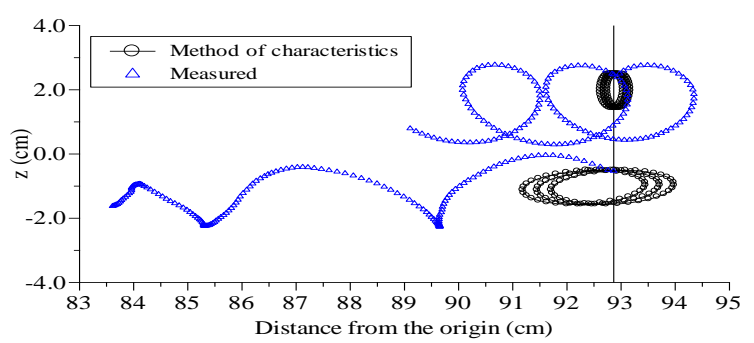

(b) $\mathrm{T}=7.2 \mathrm{~s}, \mathrm{x}=106 \mathrm{~cm}$

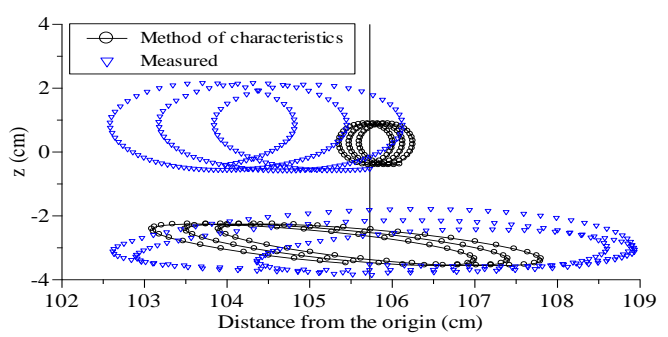

Fig. 9 Computed water particle trajectories by the method of characteristics for $h_{I}: h_{I I}=15 \mathrm{~cm}: 15 \mathrm{~cm}$ 
(a) $\mathrm{T}=5.2 \mathrm{~s}, \mathrm{x}=114 \mathrm{~cm}$

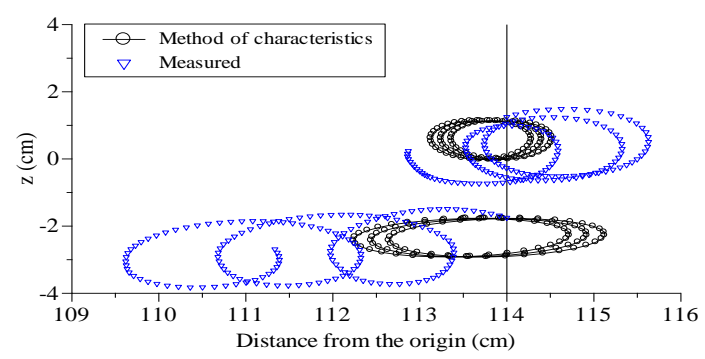

(b) $\mathrm{T}=7.2 \mathrm{~s}, \mathrm{x}=155 \mathrm{~cm}$

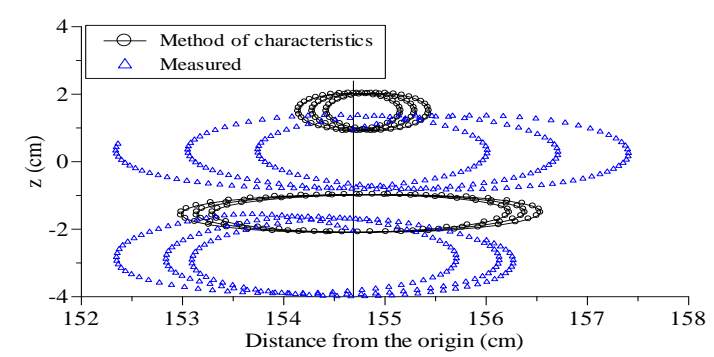

Fig. 10 Computed water particle trajectories by the method of characteristics for $h_{I}: h_{I I}=10 \mathrm{~cm}: 20 \mathrm{~cm}$

\subsection{Mass transport due to the propagation of internal waves}

The mass transport velocity can be estimated from the particle movement. It has been estimated as the ratio of horizontal particle excursion to its travel time from measured and computed data. Figs. 11 and $\mathbf{1 2}$ show the vertical distributions of averaged mass transport velocity for $h_{I}: h_{I I}=15 \mathrm{~cm}: 15 \mathrm{~cm}$ in one wave cycle. Fig. 11 (a) depicts several profiles of measured mass transport velocity for $\mathrm{T}=5.2 \mathrm{~s}$. At six locations, $\mathrm{x}=93,106,119$, $133.5,147$, and $160 \mathrm{~cm}$, all velocity profiles have a similar trend. The velocities are negative from $\mathrm{z}=-5 \mathrm{~cm}$ to $\mathrm{z}=5 \mathrm{~cm}$. It means that the internal waves tend to transport water in the direction of wave propagation near the

\section{(a) Measured}

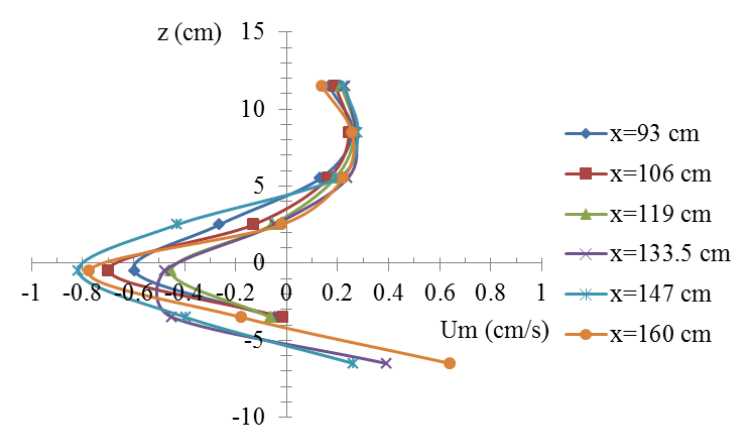

interface. However, in the remaining layers, the waves transport water in the opposed direction. The maximum value of mass transport velocities is about $0.8 \mathrm{~cm} / \mathrm{s}$. This velocity is about $20 \%-30 \%$ of the water particle velocity of internal waves. Fig. 11 (b) depicts the computed mass transport velocity for $h_{I}: h_{I I}=15 \mathrm{~cm}: 15 \mathrm{~cm}$ and $T=5.2 \mathrm{~s}$. Patterns of computed mass transport velocity show a similar trend when fitted to the corresponding measured pattern in Fig. 11 (a). The maximum velocity is approximately $0.3 \mathrm{~cm} / \mathrm{s}$. The difference between the computed and measured maximum values might be caused by many reasons. In addition to the enclosed flume problem, in the Boussinesq approximation in the 2D hydrodynamic model might cause this difference.

(b) Computed by 2D model

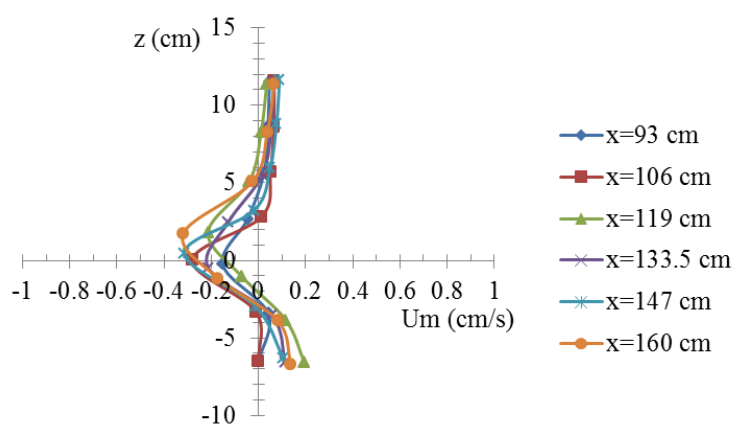

Fig. 11 Profiles of mass transport velocity for $h_{I}: h_{I I}=15 \mathrm{~cm}: 15 \mathrm{~cm}$, and T=5.2 s

(b) Computed by $2 \mathrm{D}$ model
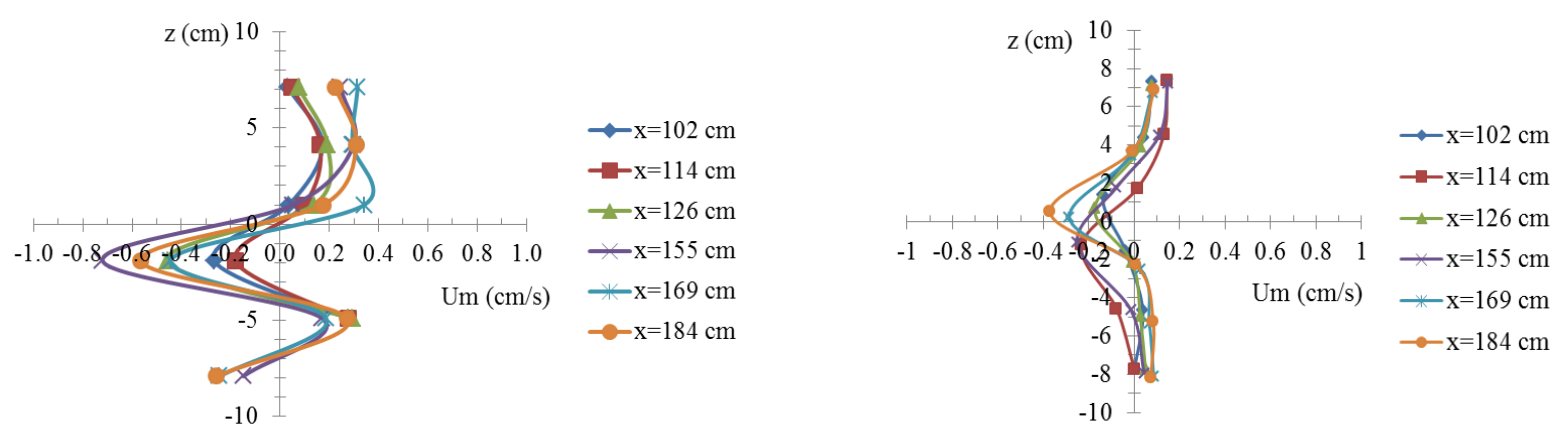

Fig. 12 Profiles of mass transport velocity for $h_{I}: h_{I I}=10 \mathrm{~cm}: 20 \mathrm{~cm}$, and T=5.2 s 
However, inspecting the measured and computed results, the measured mass transport velocity agrees with computed one on the variation along the slope.

Fig. 12 (a) depicts similar profiles of measure mass transport velocity for $\mathrm{h}_{\mathrm{I}}: \mathrm{h}_{\mathrm{II}}=10 \mathrm{~cm}: 20 \mathrm{~cm}$ with $\mathrm{T}=5.2 \mathrm{~s}$ at six elevations. In this case, the measured mass transport velocity is positive in the upper layer. These values are negative at $\mathrm{z}=1 \mathrm{~cm}$ and $\mathrm{z}=-7 \mathrm{~cm}$, but positive at $\mathrm{z}=-5 \mathrm{~cm}$ in the lower layer. Fig. 12 (b) shows the computed mass transport velocity for the corresponding locations. The computed velocity changes the elevation of maximum value along the slope. The measured and computed results confirm that the mass is transported to the shoreward direction near the density interface and to the offshore direction in the remaining layers.

\section{Conclusion}

This paper presented an investigation of water particle trajectory and mass transport of internal waves for different thickness ratios and wave periods along a uniform slope in a two-layer density-stratified fluid. The theoretical solution for water particle trajectory was derived applying the method of characteristics. Experiments were also carried out to confirm the trend of water particle movement and the result was compared to the analytical and numerical predictions. When internal waves propagate along a slope, the particles near the density interface move in the shore direction while those in the lower and upper layers move in the offshore direction. All particles march in a nonclosed loop and move clockwise and anticlockwise in the upper and lower layers, respectively. Therefore, the internal waves transport the mass with the maximum velocity in the shore direction near the density interface. The mass transport velocity decreases toward the both boundaries.

\section{REFERENCES}

1) Longuet-Higgins, M.S.: Mass transport in water waves, Philo. Trans. Royal Soc. London, Series A, Vol.
245 (903), pp. 535-581, 1953.

2) Constantin, A.: The trajectories of particles in Stokes waves, Invent. Math., 166, 523-535, 2006.

3) Constantin, A., and Villari G.: Particle trajectories in linear water waves, J. Math. Fluid Mech., 10(1), pp. 1$18,2008$.

4) Chang, H.-K., Chen Y.-Y., and Liou J.-C.: Particle trajectories of nonlinear gravity waves in deep water, Ocean Eng., 36, pp. 324-329, 2009.

5) Wunsch, C.: Note on some Reynolds stress effects of internal waves on slopes, J. Deep-Sea Res., 18, pp. 583-591, 1971.

6) Umeyama, M. and Matsuki, S.: Measurements of velocity and trajectory of water particle for internal waves in two density layers, Geophysical Res. Lett., 38, AGU, L03612, 2011.

7) Umeyama, M., Shintani, T., Nguyen, K.-C., and Matsuki, S.: Measurements of particle velocities and trajectories for internal waves propagating in a density-stratified two-layer fluid on a slope, in: Cavazzini Giovanna (Eds.), Particle Image Velocimetry, InTech, 2012.

8) Umeyama, M. and Shintani T.: Transformation, attenuation, setup and undertow of internal waves on a gentle slope, J. Waterway, Port, Coastal, and Ocean Eng., ASCE, 132 (6), 477-486, 2004.

9) Michallet, H., and Ivey G.N.: Experiments on mixing due to internal solitary waves breaking on uniform slopes, J. Geophys. Res., 104 (C6), pp. 13467-13477, 1999.

10) Walker, S.A., Martin, A.J., Easson, W.J., and Evans, W.A.B.: Comparison of laboratory and theoretical solitary wave kinematics, J. Waterway, Port, Coastal, and Ocean Eng., ASCE, 129, pp.201-218, 2003.

11) Umeyama, M.: PIV techniques for velocity fields of internal waves over a slowly varying bottom topography. J. Waterway, Port, Coastal, and Ocean Eng., ASCE, 134 (5), pp.286-298, 2008.

(Received March 8, 2012) 